\title{
Un élan missionnaire à Gaspé : les Soeurs Missionnaires du Christ-Roi (1928-1972)
}

\section{Andrée Désilets}

Volume 46, 1979

URI : https://id.erudit.org/iderudit/1007116ar

DOI : https://doi.org/10.7202/1007116ar

Aller au sommaire du numéro

Éditeur(s)

Les Éditions Historia Ecclesiæ Catholicæ Canadensis Inc.

ISSN

0318-6172 (imprimé)

1927-7067 (numérique)

Découvrir la revue

Citer cet article

Désilets, A. (1979). Un élan missionnaire à Gaspé : les Soeurs Missionnaires du Christ-Roi (1928-1972). Sessions d'étude - Société canadienne d'histoire de l'Église catholique, 46, 65-85. https://doi.org/10.7202/1007116ar

Tous droits réservés @ Les Éditions Historia Ecclesiæ Catholicæ Canadensis Inc., 1979
Ce document est protégé par la loi sur le droit d'auteur. L'utilisation des services d'Érudit (y compris la reproduction) est assujettie à sa politique d'utilisation que vous pouvez consulter en ligne.

https://apropos.erudit.org/fr/usagers/politique-dutilisation/ 


\section{Un élan missionnaire à Gaspé : \\ Les Soeurs Missionnaires du Christ-Roi (1928-1972)}

Dans une conférence intitulée Le Canada apostolique ${ }^{1}$ qu il donnait à Montréal le 5 décembre 1918, dans le but de recueillir quelques aumônes pour les Sœurs Missionnaires de l'ImmaculéeConception - communauté qui avait été fondée à Montréal en 1902 - Henri Bourassa faisait la revue des œuvres missionnaires du Canada français puis il affirmait que la caractéristique des missions canadiennes «[...] était peut-être la diversité des œuvres et la facilité d'adaptation des [. . . ] missionnaires à toutes les nècessités de l'apostolat ${ }^{2}$ ». Ces quelques mots expliquaient, avant la lettre, la fondation de la communauté des Sœurs Missionnaires du Christ-Roi et l'élan missionnaire que la communauté a suscité dans la Gaspésie pendant près de cinquante ans.

On a déjà fait l'histoire rudimentaire de la communauté des Sœurs Missionnaires du Christ-Roi. C'est l'historien Lionel Groulx qui, le premier, en a signalé les débuts et les réalisations principales dans Le Canada français missionnaire : une autre grande aventure. En 1962, Groulx donnait suite à !'œuvre de Bourassa qui, après avoir souligné les limites de sa propre synthèse, dans Le Canada apostolique, avait émis le souhait qu'elle suggère: «des études plus approfondies et des travaux plus substantiels ${ }^{3}$ ». En retraçant ce qu'il appelait «l'épopée des derniers siècles de l'histoire de l'Église et du Canada français ${ }^{4}$ », Groulx étudiait l'expansion missionnaire du Canada au pays et hors du pays, et il consacrait quelques lignes rapides, ici et là, aux Sœeurs Missionnaires du Christ-Roi, ne leur

1 Cette conférence a paru, largement amplifiée, en 1919, sous le titre Le Canada apostolique, Montréal, Bibliothèque de l'Action Française, 1919, $176 \mathrm{pp}$.

2 H. Bourassa, Le Canada apostolique, p. 8.

3 Loc. cit.

4 L. Groulx, Le Canada missionnaire: une autre grande aventure, Montréal et Paris, Fides [1962]. 534pp., p. 40. 
donnant, en toute justice, que la place qui leur revenait dans une synthèse très large. Neuf ans plus tard, soit en 1971, Sœur Louisa Poirier racontait dans la Revue d'Histoire de la Gaspésie l'histoire de la communauté en quelque huit pages qui demeurent très minces et trop modestes. Sœur Poirier exploitait alors les Annales des Ursulines de Gaspé ainsi qu'une lettre inédite de la co-fondatrice des Sœurs Missionnaires du Christ-Roi et elle citait surtout de larges extraits de ces documents ${ }^{5}$. Dans un autre numéro de la Revue d'Histoire de la Gaspésie, consacré au cinquantième anniversaire de la fondation du diocèse, on a fait place à la communauté des Sœurs Missionnaires du Christ-Roi dans un article anonyme sur les fondations religieuses du diocèse. Mais là encore, il ne s'agit que d'une partie d'un ensemble et d'une page d'histoire forcément réduite à sa plus simple expression ${ }^{6}$. Enfin, en 1978, à l'occasion du cinquantième anniversaire de fondation de la communauté elle-même, Sœur Denise Goulet. assistante générale, a publié dans l'Église canadienne un survol - plus substantiel cependant que les écrits précédents - de l'histoire de la communauté. Sœur Goulet a écrit avec intelligence. amour et ficrté. Elle présente un article aussi vivant que les sources orales qui l'inspiraient. mais son article a les limites et le caractère de l'article de circonstance ${ }^{7}$.

Après cette revue bibliographique, il m'a donc semblé qu'il y avait place pour une étude plus large sur les Sœurs Missionnaires du Christ-Roi, même si la documentation est difficile à recueillir. En effet, la fondatrice a scellé les principaux documents -- surtout ceux qui concernent les événements relatifs à la fondation - dans un coffret qu'elle a fait déposer à l'évêché de Gaspé et qui ne sera ouvert, selon ses dernières volontés, qu'en 1993, vingt-cinq ans après sa mort ${ }^{8}$. Mais les documents déjà accessibles et les témoignages recueillis dans la communauté des Sœurs Missionnaires du ChristRoi, comme dans les autres communautés missionnaires existant au

5 L. PoIrifr, "L'histoire des Sœurs Missionnaires du Christ-Roi», Revae d'Histoire de la Gaspésie, vol. X, n" 2 (avril-juin 1971). pp. 284 à 292. La co-fondatrice est sœur Antoinette Giroux, sœur de la fondatrice Frédérica Giroux.

6 AnOnyme, "L'Histoire des Fondations», Re'rue d'Histoire de la Gaspésie, vol. X. n's 2 et 3 (spécial) (avril-septembre 1972), p. 192.

7 D. Goulet, "De Gaspé en Haïti via le Japon». L'Église canadienne, vol. XII. n" 4 (19 oct. 1978). pp. 121 à 126.

8 Interview avec Sœur Denise Goulet. Maison généralice des Sœurs Missionnaires du Christ-Roi, 4730 ouest, boul. Lévesque. Chomedey, 20 juin 1979. 
Québec, notamment les Sœurs Missionnaires de l'Immaculée-Conception et les Sœurs Missionnaires de Notre-Dame-des-Anges, nous ont permis de préparer cette étude à trois volets :

- une femme et son charisme ;

- une communauté et son œuvre;

- une communauté et ses effectifs.

\section{UNE FEMME ET SON CHARISME}

La congrégation des Sœurs Missionnaires du Christ-Roi a été fondée en octobre 1928, à la suite de dissensions qui couvaient depuis une dizaine d'années dans la communauté des Missionnaires de l'Immaculée-Conception. Cette dernière communauté avait été fondée en $1902^{9}$. Elle était la première communauté missionnaire féminine fondée au Québec et elle devait en être la seule jusqu'en 1918, alors qu'était organisée la société des Sœurs Missionnaires de NotreDame-des-Anges, autre communauté issue des Missionnaires de l'Immaculée-Conception ${ }^{10}$.

La fondatrice, Frédérica Giroux ${ }^{11}$, était religieuse chez les Missionnaires de l'Immaculée-Conception depuis vingt ans quand elle mit fin à la lutte qu'elle menait depuis une dizaine d'années contre la fondatrice de la communauté sur deux questions fondamentales: la fin et la spiritualité de la communauté. Après son entréc au noviciat d'Outremont, Frédérica Giroux avait d'abord travaillé pour la communauté à Montréal et elle avait été maîtresse des novices et assistante générale pendant dix ans. En 1921, elle fondait à Vancouver une maison, sorte de pied-à-terre pour les missionnaires de Chine. Après vingt ans de vie religieuse dans une communauté missionnaire, Sæur Giroux n'était done pas encore allée en mission et elle en était profondément déçue. En récapitulant les vingt dures années qu'elle avait passées chez les Missionnaires

9 Voir Mère MArif-du-SAint-Esprit, Montréal, Maison mère des Scurs Missionnaires de l'Immaculée-Conception, [1960], 98pp.: et Mère Madeleine Lorangr:R, «Historique de la congrégation des Missionnaires de l'Immaculée-Conception et des origines de la Société des Missions étrangères du Québec ». Rapport de la SCHEC, 1971, pp. 71-84.

10 Voir Émile Grovais, Les Scrurs Missionnaires de Notre-Dame-desAnges de Sherbrooke, Québec. Bélisle, [1963], 175pp.

11 En communauté, Scur Frédérica Giroux portera le nom de Mère Marie-du-Sacré-Cœur. Mais nous la désignerons par son nom civil dans cette étude. 
de l'Immaculée-Conception, la fondatrice avait avoué à l'une de ses sœurs, en un moment de confidence : «Ce dont j'ai le plus souffert. c'est de ne pas aller en mission $12 »$. Par contre, dans ses fonctions d'assistante générale, elle avait souvent été témoin de la déception des sœurs que la communauté gardait au pays: "J'ai tellement accueilli de sœurs qui venaient pleurer parce qu'elles n'étaient pas envoyées en mission ${ }^{i 3} \gg$. Comme elle était appelée à participer aux grandes orientations de la jeune communauté, Sœur Giroux est venue en conflit avec la fondatrice sur cette question essentielle : la diversification des fins d'une communauté missionnaire et. plus concrètement, l'établissement et le soutien d'œuvres en territoire 《non infidèle ${ }^{14}$ ». Pour Frédérica Giroux. la multiplication des fins était contraire à la nature même d'une communauté missionnaire et au droit essentiel des jeunes filles qui choisissaient de servir l'Église en pays de mission. Sœur Giroux en est donc venue à projeter la fondation d'une communauté essentiellement missionnaire ${ }^{15}$. Ce sera là la caractéristique première de la Congrégation des Sœurs Missionnaires du Christ-Roi. Tous les textes ${ }^{16}$ relatifs à la fondation sont

12 Intervicw avec Scur Rollande Fournicr. Maison généralice des Sours Missionnaires du Christ-Roi.

13 Loc. cit.

14 Mère Madeleine Loranger montre bien comment l'établissement de maisons au pays (écoles apostoliques, ouvroirs, maisons de retraites fermées. hôpitaux et refuges pour Orientaux immigrés au Canada) s'insère dans un grand plan daction missionnaire qui pourrait se résumer ainsi : « [..] pour aller porter la Bonne Nouvelle aux confins du monde, il faut des apôtres. des recrues pour la relève. des ressources financières" et. en conséquence. des maisons au pays où se préparera la mission. («Historique...». Rapport SCHEC, 1971, p. 82).

15 En fait, il existait au Québec deux autres communautés missionnaires: les Franciscaines Missionnaires de Marie et les Scurs Missionnaires de Notre-Dame d'Afrique (Sœurs Blanches). implantées au pays respectivement en 1892 et en 1903, et dont la dernière, au moins. était essentiellement missionnaire. Mais ces communautés étaient, au sens fort du terme. "internationales », de sorte que la recrue renonçait à son pays d'origine et perdait en quelque sorte sa nationalité, travaillant toujours au sein dune équipe internationale. $11 \mathrm{y}$ avait donc place pour un autre type de communauté missionnaire, rattachée et identifiée au pays d'origine. À ce type correspondaient déjà les communautés des Missionnaires de l'Immaculée-Conception (1902) et des Missionnaires de Notre-Dame-des-Anges (1919). Mais ces deux communautés n'étaient pas exclusivement missionnaires.

16 Nous avons consulté ces documents aux Archives des Squrs Missionnaires du Christ-Roi (ASMCR). Les principaux sont : lre Supplique présentée en 1928 par M!ır Ross au Saint-Père: Lettre au Saint-Père pour appuýer la Supplique officielle: Première notice de la Congrégation écrite par la fondatrice en 1928 ; Décret d'érection par Mur Ross. 
bien explicites -- un peu insistants même - sur la fin unique, exclusive de la nouvelle communauté : l'extension du règne de Jésus-Christ chez les infidèles. D'après un témoin, qui a peut-être été un confident, c'est là le trait distinctif de la communauté de refuser «toute fondation d'œuvres en dehors des pays infidèles, sauf les noviciats et autres maisons de formation 17 ».

Le geste de Sœur Giroux, qui l'a amenée à quitter sa communauté pour en créer une autre, était audacieux, surtout à l'époque. Dans le contexte social du temps, doublé de la mentalité ecclésiale d'avant Vatican II, il équivalait à une apostasie et appelait le scandale. Pourtant, il n'était pas sans précédent dans l'histoire des communautés missionnaires de femmes au Québec. Neuf ans plus tôt, en 1919, une autre communauté missionnaire était issue des Missionnaires de l'Immaculée-Conception, celle des Sœurs Missionnaires de Notre-Dame-des-Anges. Sœur Florina Gervais ${ }^{18}$, née en 1888 et Missionnaire de l'Immaculée-Conception de 1906 à 1914, qui avait passé quatre ans en Chine continentale, à Canton, était venue en conflit avec sa propre communauté sur un autre point essentiel : l'intégration de jeunes Chinoises à la communauté, c'està-dire la dénationalisation des communautés missionnaires et, indirectement, l'affirmation du caractère supranational et exclusivement religieux de la mission. Sœur Gervais avait donc quitté sa communauté en 1914, était retournée trois ans en Chine puis, avec l'appui de $M^{* r}$ Paul Larocque, de Sherbrooke, elle avait organisé à Lennoxville sa propre communauté qui comprenait déjà 44 professes en 1928, dont une Chinoise ${ }^{19}$.

17 Louis Lalande, «Missionnaires du Christ-Roi », Le Messager canadien du Sacré-Caur, vol. XXXVIII, n" 3, mars 1929, pp. 110-111.

18 Dans sa communauté, Sơur Florina Gervais portera le nom de Mère Marie-du-Sacré-Cœur comme Sœur Giroux dans sa propre communauté. Mais nous la désignerons par son nom civil dans notre étude.

19 Registre des Sxurs, Archives des Sœurs Missionnaires de NotreDame-des-Anges (ASMN-D-A). Maison généralice, Lennoxville. D’après un témoin qui tenait le fait de Sœur Giroux elle-même, quand Sœur Gervais avait quitté les Missionnaires de l'Immaculée-Conception, elle aurait prédit à Sœur Giroux qui le lui reprochait : "Dans cinq ans, ce sera votre tour», ce qui laisse entendre que le climat à l'intérieur de la communauté n'était pas à son meilleur. Il faut préciser que Sœur Gervais n'avait fait que des vœux temporaires chez les MIC, le 8 septembre 1906. À ce point de vue, son engagement dans la communauté était différent de celui de Sœur Giroux qui avait fait profession perpétuelle le 11 février 1920 et qui n’a pu quitter sa communauté qu'avec un indult pontifical. 
Les deux nouvelles fondations répondaient non seulement au charisme et au dynamisme de deux femmes mais aussi à l'attente de l'Église qui repensait alors son idéal missionnaire, revisait sa conception de la mission, procédait à l'étude scientifique des missions, développait ses activités missionnaires et renouvelait ses méthodes d'évangélisation.

Après la Première Guerre mondiale, qui avait nécessairement marqué un temps d'arrêt dans le recrutement et l'action missionnaires, Benoit XV publiait son encyclique Maximum Illud le 30 novembre 1919, qui affirmait d'abord et surtout le caractère supranational des missions ${ }^{20}$ et expliquait la nécessité de former et d'organiser un clergé indigène, ce qui signifiait aussi, bien entendu. des religieux et des religieuses autochtones. Benoit XV écrivait: « $(\ldots)$ le prêtre indigène que tout, naissance, mentalité, impressions, idéal, rattache à ses ouailles, est merveilleusement armé pour acclimater la vérité dans les âmes : bien mieux que tout autre, il sait choisir les moyens de forcer la porte des cœurs. C'est ainsi qu'il a facilement accès auprès de bien des âmes dont le prêtre étranger se voit interdire le seuil ». Et Benoît XV insistait pour que les indigènes ne soient plus destinés «à servir d'auxiliaires aux missionnaires étrangers dans les fonctions les plus humbles du ministère », au contraire, qu' «ils soient mis à même de prendre un jour en main la direction de leurs ouailles». Tel devait être l'idéal de l'Église missionnaire selon Benoît XV. Pie XI, pour sa part, s'identifia tellement à l'Église missionnaire qu'il se mérita le titre de "Pape des missions». Dans son encyclique Rerum Ecclesiae du 18 février 1926, il exposa à son tour les raisons théologiques, canoniques, pastorales, voire politiques, d'établir «stablement » l'Église dans les missions, en lui donnant «tous les éléments»d'une Église constituée, soit un clergé, un peuple propre à chaque région ainsi que des religieux de l'un et de l'autre sexe ${ }^{21}$.

Mais à mesure que l'Église développait, précisait et énonçait sa pensée missionnaire, il était normal que les conceptions de la

$20 \mathrm{Au}$ XVI' siècle, l'Église s’était appuyée sur le Portugal et l'Espagne pour accomplir son œuvre apostolique ; au XIX" siècle, elle avait compté sur la France et l'Autriche. Désormais, elle s'appuierait, d'une part, sur Rome où seraient concentrées les ressources missionnaires et, d'autre part, sur le peuple chrétien qui serait sollicité pour ses ressources humaines et financières.

21 Voir Georges Goyau, Missions et Missionnaires, Bloud \& Gay, [1931], 272pp. 
mission se diversifient ${ }^{22}$, tous les missionnaires ne pouvant assimiler les messages pontificaux au même rythme. Il était donc normal que les deux fondatrices des Sœurs Missionnaires du Christ-Roi et des Sœurs Missionnaires de Notre-Dame-des-Anges répondent à une inspiration qui leur était venue de l'expérience et qui s'insérait dans les vœux des dernières et grandes encycliques sur les missions. Pour elles, le message ecclésial était clair et elles ne faisaient que l'actualiser. En théorie, leurs initiatives auraient $\mathrm{pu}$ se réaliser dans le cadre de la communauté des Missionnaires de l'ImmaculéeConception dont elles étaient membres. Mais en pratique, ce fut l'affrontement de personnalités fortes et tenaces (faudrait-il dire 《illuminées»), et les ruptures devinrent inévitables, ce qui risquait de discréditer les dissidentes auprès de l'épiscopat.

Pourtant, Sœur Giroux eut d'abord l'appui de $\mathbf{M}^{\mathrm{ar}}$ Guillaume Forbes, de Joliette, qui aurait sans doute accueilli la nouvelle communauté dans son diocèse s'il n'avait été lui-même muté à Ottawa. Il confia alors le projet à $\mathrm{M}^{\mathrm{gr}}$ Ross qui était le seul évêque qui n'avait pas de communauté missionnaire dans son diocèse et dont l'esprit missionnaire était reconnu ${ }^{23}$. $\mathbf{M}^{\mathrm{wr}}$ Ross avait rêvé autrefois aux missions de la Côte-Nord, il avait sollicité en vain de son évêque l'autorisation de se vouer aux missions, fondé à Rimouski une école apostolique pour jeunes filles et favorisé les vocations missionnaires dans son clergé depuis la fondation du diocèse de Gaspé en 1923, en dépit de la pénurie de sujets ${ }^{24}$. Malgré son zèle pour toute entreprise missionnaire, $\mathrm{M}^{\mathrm{gr}}$ Ross accueillit prudemment Sœur Giroux, vu les conditions particulières dans lesquelles le projet avait été conçu. En fait, dans une lettre au Saint-Père, qui appuyait la supplique officielle pour obtenir l'autorisation de commencer à Gaspé la fondation des Sœurs Missionnaires du Christ-Roi, M ${ }^{\text {ar }}$ Ross écrivit: " Je ne puis taire que c'est après un refus catégorique, puis de longues hésitations, que je me suis rallié à l'idée de fonder une congrégation nouvelle s'ajoutant à tant d'autres qui existent déjà. Il s'ajoutait à cette première objection une autre source d'appréhension, à savoir que la Religieuse qui me demandait de patronner son projet $[\ldots]$ pouvait se laisser guider par des motifs d'ordre

22 Il faut noter que le premier Institut de Sciences missionnaires canadien ne fut créé qu'en 1948, à l'Université d'Ottawa. ASMCR.

$23 \mathrm{M} \mathrm{rr}^{\mathrm{r}}$ Ross, Lettre au Saint-Père pour appuyer la Supplique officielle,

24 M $*$ Ross, op. cit.. p. 1, par. 5. Voir aussi Michel Lemoignan, «La vision audacieuse de M*r F.-X. Ross, premier évêque de Gaspé »,Rapport de la SCHEC, 1976, pp. 35 à 47. 
temporel et mettre en avant ce projet de fondation comme un prétexte d'échapper aux obligations de sa propre Congrégation 25 ». Mais après l'épreuve du temps qui mit en relief l'authenticité du projet et la sincérité de la fondatrice, $\mathbf{M}^{* r}$ Ross ouvrit son diocèse à Sœur Giroux, l'appuyant fermement dans toutes les démarches préliminaires - spirituelles, matérielles et canoniques - à la fondation d'une nouvelle communauté ${ }^{26}$. Ainsi, les principes de Sœur Giroux trouvaient le cadre nécessaire à leur application ct une communauté missionnaire à fin unique était fondée à Gaspé ${ }^{27}$.

Lorsqu'elle était encore religieuse chez les Missionnaires de I'Immaculée-Conception, Sœur Giroux avait été en désaccord avec la fondatrice sur une dcuxième question essentielle : la spiritualité de la communauté. En lui donnant son nom, Société des Sœurs Missionnaires de l'Immaculée-Conception ${ }^{28}$, le pape Pie $\mathrm{X}$ avait

25 M $*$ r Ross. Lettro au Saint-Père pour appuyer la Suppliqui officielle. p. 1, par. 2. ASMCR. Les faits contredisent donc linterprétation de Sœur Marguerite Jean qui attribue à $M * r$ Ross toute l'initiative de la fondation et un simple rôle d’assistance à Sœur Giroux. Sœur Jean écrit : «Les Sœurs Missionnaires du Christ-Roi [furent] fondées à Gaspé en 1928 par Morr François-Xàvier Ross. [... C'esi une sceur missionnaire de l'Immaculée-Conception, Frédérica Giroux qui [...] en sortit pour aider la fondation gaspésienne et devenir la première supérieure de la communauté ». Voir Sœur Marguerite JEAN, Érolution des communautés religieuses de femmes au Canada de 1639 à nos jours, Montréal, Fides, [1977], 324pp., p. 163.

26 Dans sa Lettre alt Saint-Père..., p. 2, au paragraphe intitulé Fondutcur, $\mathrm{M} * \mathrm{r}$ Ross se dit «prêt à assumer la responsabilité morale» de la fondation et «à en prendre la haute direction», ASMCR. Il est donc clair que les communautés religieuses sont sous la direction et la responsabilité d'autorités masculines : "l'évêque du lieu». si la communauté est de droit diocésain; et la Sacrée Congrégation des Religieux, si elle est de droit pontifical. Ce fait contredit donc une interprétation féministe de l'histoire, qui voudrait que les religieuses aient été les premières féministes du Québec. Voir Micheline Dumont-Johnson. «Les communautés religieuses et la condition féminine». Recherches sociographiques, vol. XIX, n"1, janvier-avril 1979. Dans cet article, plus sociologique qu'historique, l'auteur néglige le droit canon et toute spiritualité : elle ignore donc la régie et la vie internes des communautés religieuses.

27 La fondation officielle de la communauté des Scurs Missionnaires du Christ-Roi est d'octobre 1928. L'érection canonique est du 11 février 1930 : l'incorporation civile. du 11 mars 1931 ; le bref de louange qui l'érige en Congrégation de droit pontifical. le 7 mars 1963. ASMCR.

28 Voir Mère Marie'dlu-Saint-Esprit, p. 44. Pie X aurait dit à M ${ }^{g r}$ Bruchési qui lui demandait conseil au sujet de la fondation projetée : «Fondez, fondez. toutes les bénédictions du Ciel descendront sur le nouvel Institut auquel vous donnerez le nom de Société des Sœurs Missionnaires de l'Immaculée-Conception :. 
nettement orienté la première communauté missionnaire du Québec vers une spiritualité mariale, et la fondatrice développait particulièrement cette dominante. Ainsi, elle s'adonna à la propagation de la dévotion mariale selon Louis-Marie-de-Montfort et fit du Rosaire un exercice privilégié ${ }^{29}$. Pour sa part, Sœur Giroux, marquée par la spiritualité ignatienne, était portée vers une spiritualité christocentrique et elle aurait voulu mettre l'Office divin, chanté et psalmodié, au cœur de la prière de la famille religieuse. Bref, deux écoles de spiritualité s'étaient affrontées au sein des Missionnaires de l'Immaculée-Conception, et l'affrontement s'était accentué à mesure que les liens intimes avec la Société des Missions Étrangères ${ }^{30}$ faisaient triompher la spiritualité sulpicienne auprès de la fondatrice des Missionnaires de l'Immaculée-Conception.

On comprend donc qu'une fois l'autorisation accordée de fonder une nouvelle communauté missionnaire, Sœur Giroux ait opté pour une appellation qui en signifiait la spiritualité christocentrique ${ }^{31}$. Rappelons que trois ans plus tôt, en 1925, Pie XI avait institué la fête du Christ-Roi, n'entrevoyant que dans le règne spirituel du Christ l'unité et la paix d'un monde bouleversé par les révolutions et les guerres. De plus, Sœur Giroux donna à la vie active de ses missionnaires l'assise d'une vie contemplative s'exprimant par l'adoration eucharistique, la dévotion au Cœur de Jésus et l'Office divin ${ }^{32}$ et fit de sa communauté la seule communauté missionnaire féminine $\mathrm{du}$ pays dont le gouvernement et les règles s'inspiraient de la Compagnie de Jésus.

29 Voir Mère Madeleine LoRANGer, "Historique de la Congrégation des...», Rapport de la SCHEC, 1971, p. 74. Notons que le vœu d'esclavage à Marie était au centre de la doctrine de Louis-de-Montfort. Voir le Traité de la Vraie Dérotion à la Sainte-Vierge.

30 La fondatrice des Missionnaires de l'Immaculée-Conception, Sœur Délia Tétrault, avait aussi collaboré à la fondation de la Société des Missions Étrangères du Québec. sur le modèle de Paris, pour prêtres qui désiraient se consacrer aux missions lointaines. Voir Mère Madeleine LoraNGER, «Historique de la Congrégation des Missionnaires de l'ImmaculéeConception ...». Rapport de la SCHEC, 1971, pp. 75 à 81.

31 Sœur Giroux aurait voulu dédier la communauté au Divin Cœur alors que $\mathbf{M}^{\mathrm{r} r}$ Ross préférait le patronage du Christ-Roi. C'est le Pape Pie XI qui trancha la question en faveur du Christ-Roi . . et de $\mathrm{M}^{\mathrm{ar}}$ Ross. Voir Lettre aıı Saint-Père.... p. 1, par. 2, ASMCR.

32 La Société des Saurs Missionnaires du Christ-Roi, première notice écrite par la fondatrice en 1928, ASMCR. 


\section{UNE COMMUNAUTÉ ET SON CEUVRE}

Fidèle à son charisme, Sœur Giroux donna donc à l'Église du Québec sa première communauté féminine exclusivement missionnaire, dont l'esprit rejoignait bien les enseignements pontificaux du temps et annonçait, de loin, le ressourcement spirituel des années 1960. Plus immédiatement, elle canalisa des énergies missionnaires en attente et, dès 1933, elle amena des Gaspésiennes à participer à l'œuvre apostolique de l'Église. Quatre missionnaires du Christ-Roi firent partie du contingent formé de 47 religieux et religieuses de diverses communautés canadiennes, qui s'embarquèrent le 17 septembre 1933 pour l'Extrême-Orient ${ }^{33}$. Ces quatre missionnaires s'établirent au Japon. Un deuxième groupe conduit par la fondatrice elle-même les rejoignit en $19377^{34}$. La volonté des Sœurs de s'implanter définitivement au Japon s'cxprima de deux façons. Dès leur arrivée, à la demande d'un missionnaire français, clles furent en charge de jeunes sœurs détachées d'une communauté japonaise, puis elles fondèrent un noviciat à la veille même de la guerre mondiale, en 1938. Cette initiative leur valut, en temps de guerre, d'être emprisonnées en résidence surveilléc plutôt qu'en camp de concentration, comme les Sœurs des autres communautés étrangères ${ }^{35}$.

Après le temps mort de la Deuxième Guerre mondiale, les œuvres se multiplièrent au Japon: un orphelinat (1946), une école maternelle (1947), une léproserie (1947) à laquelle on ajouta un hôpital général (1948) et un sanatorium (1948). Après 1960, les Sœurs répondirent aussi à des besoins nouveaux : école de langues (1962), réhabilitation des lépreux (1963) et éducation aux enfants retardés (1965).

33 Voir larticle de Sceur MARIT-DE-ST-JACques, «Gaspésiennes au Japon», Re'ule d'Histoire de la Gaspésie, vol. III, n" 1 (janvier-mars 1965), pp. 23-32.

34 Pour l'histoire de l'extension des Sœurs Missionnaires du Christ-Roi, voir Snur Denise Goulit, «De Gaspé en Haïti via le Japon », Église canadienne, vol. XII, n" 4 (19 octobre 1979), pp. 721-726. Nous avons recueilli d'autres informations dans les deux publications missionnaires que la communauté a publiées successivement: le Petit Missionnaire, bulletin d'éveil missionnaire et organe local de l'CEuvre Pontificale de la Sainte-Enfance, publié par les SMCR tous les deux mois pendant l'année scolaire de 1946 à marsavril 1960; et La Moisson, revue missionnaire publiée de janvier 1960 à décembre 1967 à raison de cinq numéros par année.

35 En 1939. le noviciat comptait 5 novices, mais une seule y est demeurée pendant la guerre. 
Mais l'œuvre au pays du Soleil-Levant ne représente qu'un chapitre de l'aventure des Sœurs Missionnaires du Christ-Roi. Déjà, pendant la guerre, en 1943, elles avaient accepté une mission auprès des déportés japonais à Kaslo et à Sandon, en Colombie britannique, et ce service les avait amenées en 1944 à ouvrir des missions chez les Amérindiens du Canada. Elles devaient travailler dans la réserve d'Anaham (C.B.) et y diriger, notamment, une congrégation autochtone ${ }^{36}$. Bientôt, elles étendirent leurs œuvres apostoliques : catéchèse, pastorale, services sociaux et services hospitaliers aux missions amérindiennes de Mount Currie (C.B. ), Anahim Lake (C.B.), Chemainus (C.B.) et Longlac (Ontario). Puis, en 1953, cinq Sœurs Missionnaires du Christ-Roi rejoignirent les quelque 900 missionnaires canadiens-français qui missionnaient en Afrique, ce continent étant devenu le plus vaste champ d'évangélisation de l'Église. Ce fut le Congo belge qui les accueillit, et on leur confia tout un éventail d'œuvres traditionnelles comme les écoles (1954), les dispensaires (1954), les hôpitaux et les maternités (1956), ou plus nouvelles comme une « œuvre des fiancés » (1954). un foyer social et des visites à domicile (1955), etc. Au Congo, devenu le Zaïre, les Sœurs Missionnaires du Christ-Roi fondèrent lcur deuxième noviciat missionnaire en 1963. et la première Zaïroise fit profession en 1966.

Et ce fut l'Amérique latine en 1975. La congrégation accepta alors de travailler à Haïti et, fait tout à fait remarquable, des Sœurs japonaises firent alors partie du groupe des premières Sœurs Missionnaires du Christ-Roi à Haiti. C'était une primeur dans l'histoire du mouvement missionnaire du Canada et un tournant dans l'histoire de la Congrégation: les évangélisées devenaient évangélisatrices, et l'œuvre presque cinquantenaire continucrait sous un visage nouveau, plus catholique, au sens étymologique du terme. C'était aussi la pleine réalisation de l'œuvre missionnaire à laquelle la fondatrice avait sans doute rêvé.

\section{UNE COMMUNAUTÉ ET SES EFFECTIFS}

Mais une communauté ne vit pas seulement de lidéal d'une fondatrice. Il lui faut la réponse d'individus. Il nous paraît donc intéressant d'ajouter à notre étude un troisième volet sur le recru-

36 Fondée en 1945, la communauté autochtone des Sceurs de MarieImmaculée disparut en 1954. 

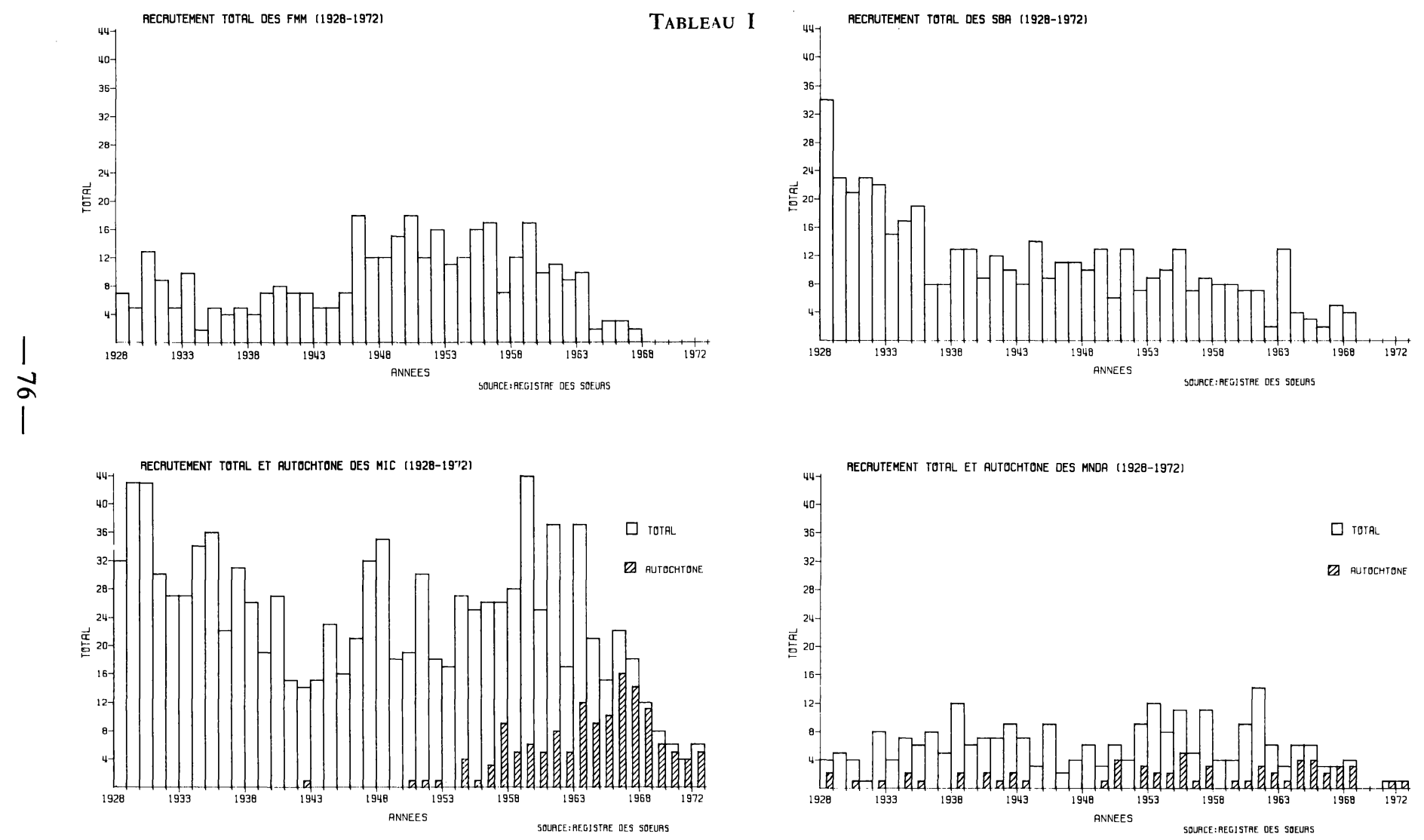
Tableau II

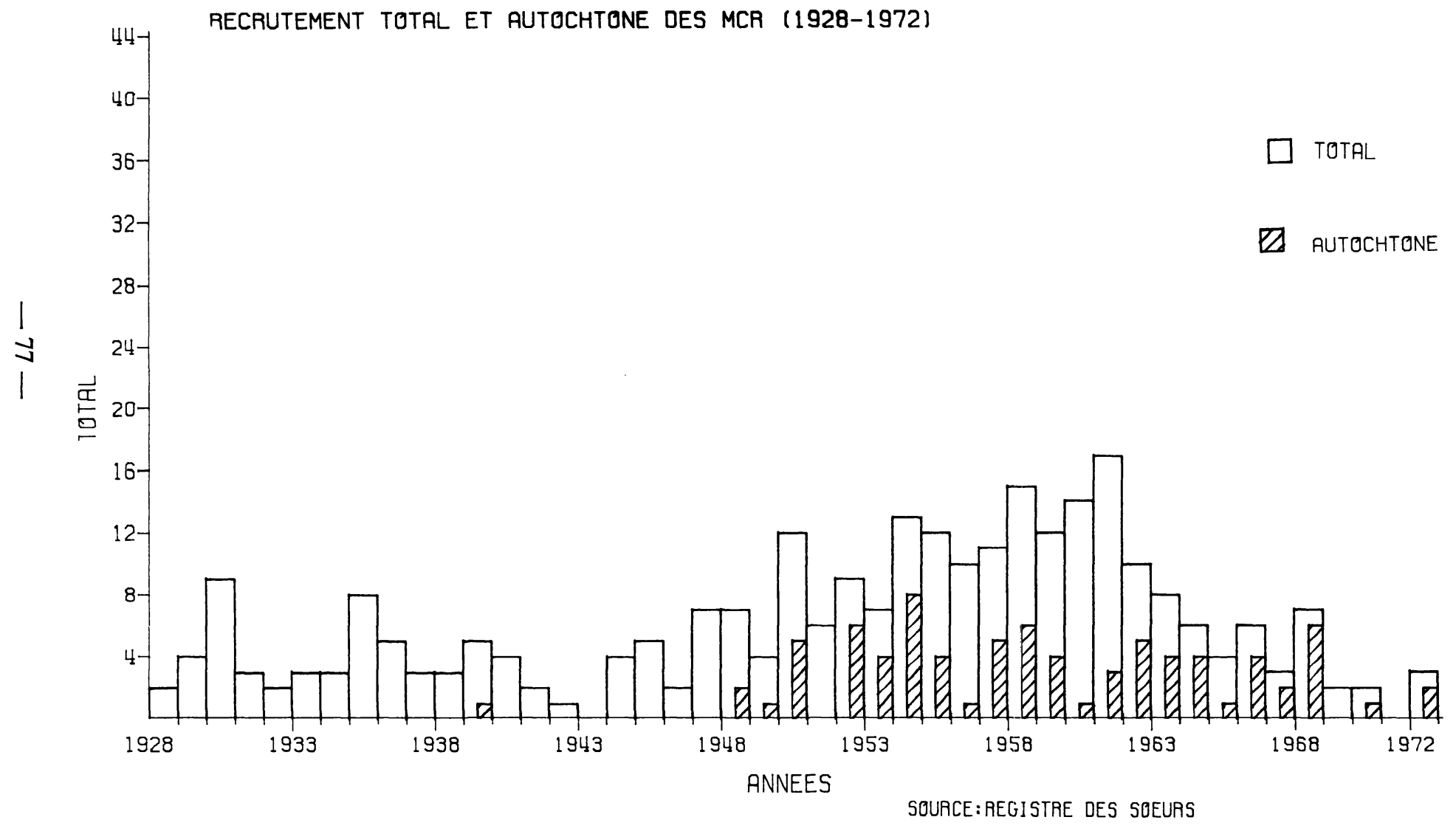


Tableau III

RECRUTEMENT TOTAL DES CING COMMUNAUTES (1928-1972)

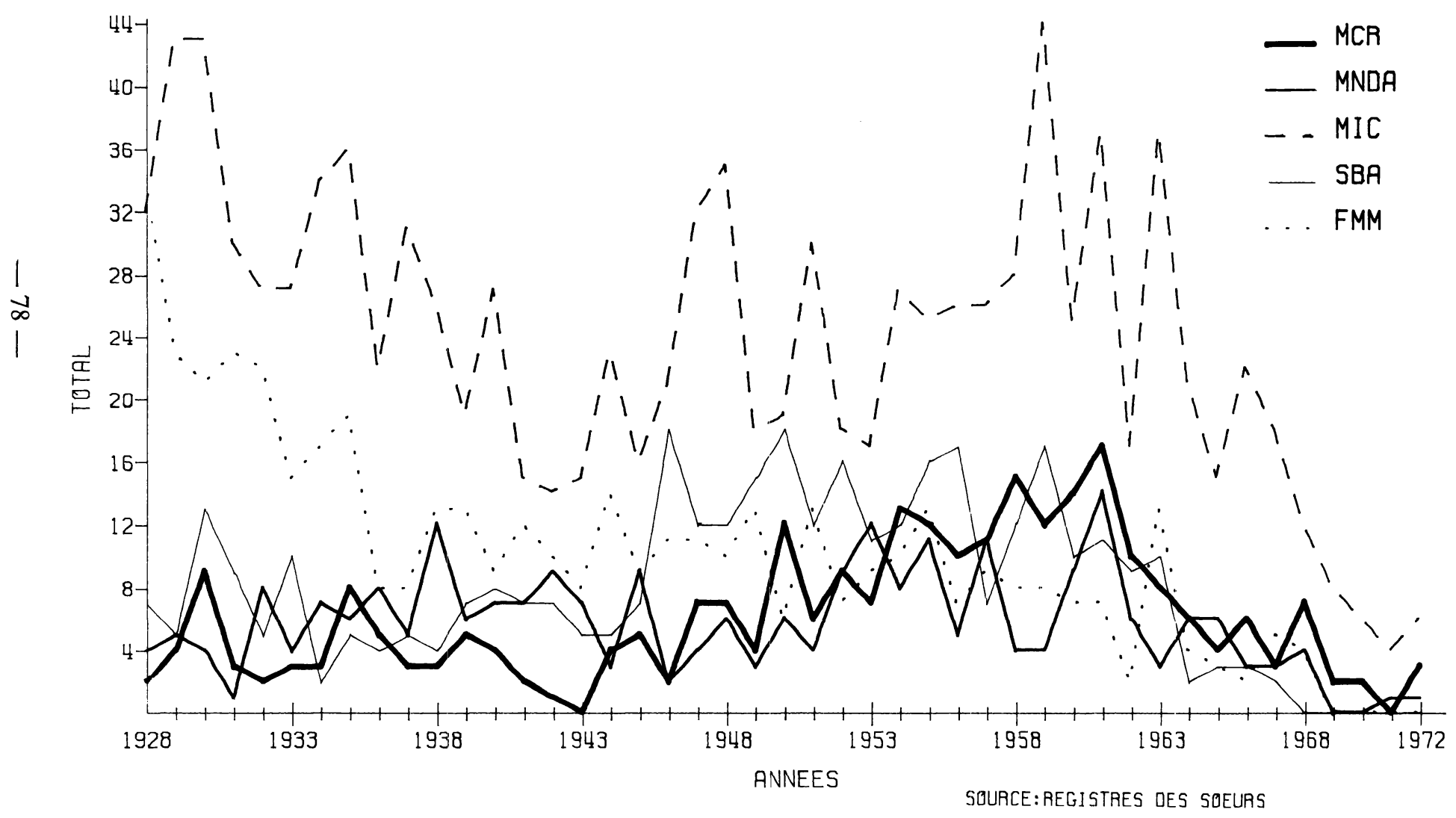


tement de la communauté. Nous analyserons le recrutement des Sœurs Missionnaires du Christ-Roi en lui-même et en comparaison $\mathrm{du}$ recrutement des quatre autres communautés missionnaires féminines qui se développaient concurremment au Québec : les Franciscaines Missionnaires de Marie (FMM) ${ }^{37}$, les Sœurs Missionnaires de Notre-Dame d'Afrique (Sœurs Blanches, SBA), les Missionnaires de l'Immaculée-Conception (MIC) et les Sœurs Missionnaires de Notre-Dame-des-Anges (MNDA). Notre documentation, que nous présentons dans les tableaux qui accompagnent notre texte, provient des registres des sœurs des cinq communautés ${ }^{38}$ pour la période allant de 1892, date d'implantation des FMM au Québec ${ }^{39}$, à 1972, alors que le recrutement canadien est presque tari dans l'une et l'autre des cinq communautés ${ }^{40}$.

$\mathrm{Au}$ moment où les Sæurs Missionnaires du Christ-Roi entrent en scène, en 1928, le recrutement missionnaire est en progression. En fait, il atteindra son sommet en 1930 , avec 90 recrues, nombre dont il ne se rapprochera qu'en 1959 et 1961 avec 85 et 86 recrues.

37 Ce sont les missions qui constituent le champ d'activité principal de cette communauté, selon son nom d'ailleurs. Il y a donc une erreur dans la Sociographie généralc des communautés religieuses au Québec de Bernard Denault, qui indique que c'est l'éducation.

38 Dans ces registres sont consignés les noms et les étapes de la vie religieuse des recrues qui ont fait une première profession dans la communauté. Notre étude ne comprend donc pas les recrues qui ont quitté la communauté avant la profession, mais elle comprend celles qui sont mortes ou qui ont quitté la communauté après les vœux temporaires. On trouve ces registres à :

1) Maison provinciale des FMM, 145. rue Pressland,

Ottawa.

2) Maison provinciale des SBA, Boucherville.

3) Maison généralice des MIC, 314. Côte Sainte-Catherine, Outremont.

39 Nous avons vu qu'une Canadienne était entrée chez les Sœurs Blanches en 1885 et trois en 1896. Mais nous retenons 1903 comme date d'implantation de la communauté au Canada, qui est donc postérieure à celle des FMM.

40 Après la Deuxième Guerre mondiale, d'autres communautés missionnaires s'implanteront au Québec: en 1953, les Sœurs Missionnaires du Saint-Esprit; en 1955, la Congrégation de Notre-Dame-des-Apôtres et, en 1957, les Sœurs Dominicaines Missionnaires de Namur. Voir Bernard Denault, Sociologic ..., pp. 195-200. Mais ces trois communautés, n'ayant qu'un nombre total restreint $(3,35,3)$ de membres en 1969 , ne peuvent offrir de points de comparaison avec les MCR. 
Des quatre communautés qui se recrutent alors au Québec, deux dominent nettement: les Franciscaines Missionnaires de Marie et les Missionnaires de l'Immaculée-Conception ${ }^{41}$. Les Sœurs Missionnaires du Christ-Roi rejoignent rapidement les deux petites communautés des Sœurs Missionnaires de Notre-Dame-d'Afrique et des Sœurs Missionnaires de Notre-Dame-des-Anges, et leur recrutement se maintient jusqu'à la guerre, sans subir les contrecoups de la crise économique, comme les grandes communautés qui accusent alors une baisse considérable de leurs effectifs ${ }^{42}$, passant entre 1930 et 1936, de 43 à 22 sœurs, pour les Missionnaires de l'ImmaculécConception, et de 21 à 8 pour les Franciscaines Missionnaires de Marie.

Pendant la guerre, la communauté doit réduirc ses activités au Japon, seul pays de mission où elle est implantée en 1939. Son recrutement s'en ressent, suivant en cela le mouvement général. D'ailleurs, le climat d'une société en guerre est tel que les jeunes diffèrent alors leur décision d'avenir ou s'orientent vers d'autres sphères d'activité humaine et sociale. Mais, avec la fin de la guerre, la reprise d'un recrutement autochtone ${ }^{43}$, l'excédent de la population féminine dû à la guerre, l'entrée en bloc des vocations différées par les conditions de guerre, la progression démographique normale et la poussée de la foi des années 1950 au Québec, la communauté accuse une augmentation graduelle de ses effectifs, comme l'ensemble des autres communautés missionnaires ${ }^{44}$.

Il faut ici mettre en relief l'apport du recrutement autochtone de la communauté. Parfois, comme en 1952 et 1953, et après 1965,

41 Les FMM ont accueilli 575 recrues entre 1892 et 1928 ; les MIC, 242 recrues entre 1902 et 1928 ; les SBA, 96 recrues entre 1903 et 1928 ; et les MNDA, 49 recrues entre 1919 et 1928. Voir Appendice.

42 Au sujet des incidences de la crise sur le recrutement des communautés religieuses, nous n'arrivons pas à la même conclusion que Marc-André LESSARd et Jean-Paul Montminy. "Les religieuses du Canada : âge, recrutement et persévérance ", Recherches sociographiques, vol. VIII, n“ 1, janvieravril 1967. pp. 15-49. Mais cette étude couvre toutes les communautés religieuses canadiennes. dont les communautés missionnaires ne constituent qu'une partie pouvant constituer une exception.

43 En 1947, les SMCR ont réouvert leur noviciat au Japon. À noter que nous désignons du terme «autochtone» la recrue dun pays de mission.

44 Les FMM font exception. Depuis 1930, dailleurs, elles étaient en constante régression, ce qui pourrait s'expliquer par l'évolution des œuvres de la communauté. Venues au Canada "en pays de mission». les FMM se situent avec le temps dans les mêmes champs d'action que beaucoup d'autres communautés canadiennes. 
il permet le maintien des effectifs de la communauté ; parfois, comme en 1950 et 1954, il permet une augmentation sensible de ces effectifs. Ce phénomène est évidemment le résultat du recrutement de 30 Japonaises entre 1948 et 1954, et de 8 Japonaises et 5 Zaïroises entre 1965 et 1968 .

Cependant, le recrutement autochtone n'y est pour rien dans les sommets qu'atteint le recrutement total en 1958, 1960 et 1961. Il faut attribuer cette hausse des vocations aux conditions idéales déjà décrites pour les années 1950 et, en partie, à l'ouverture d'un postulat à Moncton (N.-B.), qui donne 29 recrues entre 1955 et 1961. Mentionnons trois autres facteurs qui auraient joué en faveur du recrutement : l'accès plus généralisé de la jeune fille des milieux rural et ouvrier aux Écoles Normales, aux Instituts familiaux et aux Écoles d'infirmières ${ }^{45}$; une première ouverture au Tiers-Monde ${ }^{46}$; enfin, la baisse des vocations pour les œuvres traditionnelles de l'enseignement, de l'hospitalisation et des services sociaux ${ }^{47}$.

Après avoir atteint son plus haut sommet en 1961, le recrutement des Sœurs Missionnaires du Christ-Roi décroît rapidement jusqu'en 1965, alors que le déclin devient chronique, suivant la courbe générale à la baisse. En fait, l'élan missionnaire canadien est alors passé, mais il se perpétue dans l'élan missionnaire autochtone qu'il a engendré et les communautés missionnaires canadiennes présentent désormais les traits exotiques de leurs recrues africaines, asiatiques et latino-américaines.

À son apogée, l'élan missionnaire canadien avait couvert tout le Québec. Les Franciscaines Missionnaires de Marie et les Sours Missionnaires de Notre-Dame-d'Afrique avaient drainé les vocations

45 Il s'agit d'études de niveau intermédiaire par rapport aux études universitaires, et d'études faites dans des maisons religieuses, presque exclusivement des pensionnats. L'accès aux études supérieures produira l'effet contraire.

46 La même remarque vaut pour l'ouverture au tiers-monde. Une première sensibilisation suscite des vocations missionnaires tandis qu'une connaissance plus approfondie amène un autre type d'engagement (laïcat missionnaire et service dans les organismes internationaux) et même certaines défections dans les communautés missionnaires.

47 Ces facteurs expliqueraient, entre autres, les poussées exceptionnelles du recrutement des MIC au début de la période de laïcisation des années 1960. 
de la région de Québec ${ }^{48}$. Les Missionnaires de l'Immaculée-Conception, pour leur part, avaient rayonné de Montréal vers Chicoutimi et Rimouski ${ }^{49}$. Après 1919, les Cantons de l'Est avaient aussi eu leur communauté missionnaire avec les Sœurs Missionnaires de Notre-Dame-des-Anges ${ }^{50}$. Pour leur part, les Sœurs Missionnaires du Christ-Roi avaient atteint une région périphérique, jusque-là intouchée par l'appel missionnaire ${ }^{51}$. Elles avaient recruté plus de $60 \%$ de leurs effectifs en Gaspésie et en Acadie ${ }^{52}$, recueillant ainsi les vocations en attente que les communautés plus anciennes n'avaient pu atteindre. En s'implantant cn Gaspésie et en faisant de la région un foyer de rayonnement missionnaire, l'œuvre de Sœur Giroux avait donc participé à la marche de l'Église qui se fait essentiellement par enracinements et déracinements successifs.

\section{Andréc Désilets, \\ Département d'histoire, Université de Sherbrooke}

48 Les Archives des Franciscaines Missionnaires de Marie étant à leur maison généralice de Rome, nous n'avons pas pu établir l'origine géographique des Sœurs de cette communauté. Mais s'étant établies d'abord à la Baie SaintPaul puis à Québec et s'étant répandues dans la région de Qućbec, elles se sont recrutées surtout dans cette région, d'après les témoignages recueillis. Quant aux Sœurs Missionnaires de Notre-Dame d'Afrique, 61\% de leurs recrues canadiennes sont originaires de la région de Québec.

49 Les Missionnaires de l'Immaculée-Conception se sont recrutées majoritairement dans ces trois villes. Mais elles ont couvert tout le Québec et, à ce point de vue, elles pourraient constituer la seule communauté missionnaire «provinciale ».

50 Des 171 recrues québécoises, 102 sont des Cantons de l'Est, soit $60 \%$.

51 En relevant la provenance géographique des sœurs des autres communautés, nous constatons que les Gaspésiennes y sont peu nombreuses : 7 chez les MIC, 9 chez les SBA, 2 chez les MNDA.

52 Entre 1928 et 1972, la communauté a recruté 280 sœurs, dont 193 Canadiennes et 87 Autochtones ( 76 Japonaises, 10 Zaïroises et une Haïtienne). De ce nombre, 61 sont de la Gaspésie et 41 du Nouveau-Brunswick acadien qui peut être considéré dans le cas présent comme un prolongement de la Gaspésie. 
A PPENDICE 1

RECRUTEMENT TOTAL

(1892-1928)

\begin{tabular}{|c|c|c|c|c|c|c|}
\hline Années & FMM & SBA & MIC & MNDA & MCR & Total \\
\hline \multicolumn{7}{|l|}{1892} \\
\hline 1893 & 7 & & & & & 7 \\
\hline 1894 & 7 & 3 & & & & 10 \\
\hline 1895 & 11 & & & & & 11 \\
\hline 1896 & 13 & & & & & 13 \\
\hline 1897 & 15 & & & & & 15 \\
\hline 1898 & 15 & & & & & 15 \\
\hline 1899 & 16 & & & & & 16 \\
\hline 1900 & 18 & & & & & 18 \\
\hline 1901 & 9 & & & & & 9 \\
\hline 19() 2 & 27 & & 2 & & & 29 \\
\hline 1903 & 19 & 1 & 0 & & & 20 \\
\hline 1904 & 17 & 3 & 4 & & & 24 \\
\hline 1905 & 6 & 1 & 0 & & & 7 \\
\hline 1906 & 12 & 2 & 8 & & & 22 \\
\hline 1907 & 18 & 6 & 1 & & & 25 \\
\hline 1908 & 10 & 4 & 4 & & & 18 \\
\hline 1909 & 10 & 0 & 3 & & & 13 \\
\hline 1910 & 23 & 3 & 6 & & & 32 \\
\hline 1911 & 23 & 5 & 4 & & & 32 \\
\hline 1912 & 13 & 13 & 9 & & & 35 \\
\hline 1913 & 27 & 7 & 8 & & & 42 \\
\hline 1914 & 29 & 4 & 8 & & & 41 \\
\hline 1915 & 16 & 3 & 7 & & & 26 \\
\hline 1916 & 14 & 5 & 5 & & & 24 \\
\hline 1917 & 27 & 0 & 7 & & & 34 \\
\hline 1918 & 23 & 4 & 5 & & & 32 \\
\hline 1919 & 26 & 3 & 3 & $2(1)$ & & 34 (1) \\
\hline 1920 & 19 & 2 & 5 & 6 & & 32 \\
\hline 1921 & 17 & 2 & 11 & 3 & & 33 \\
\hline 1922 & 25 & 4 & 15 & 6 & & 50 \\
\hline 1923 & 18 & 1 & 19 & 7 & & 45 \\
\hline 1924 & 20 & 4 & 17 & 6 & & 47 \\
\hline 1925 & 25 & 2 & 24 & 9 & & 60 \\
\hline 1926 & 37 & 6 & 33 & 5 & & 81 \\
\hline 1927 & 31 & 7 & 34 & 5 & & 77 \\
\hline
\end{tabular}

N.B. L.es chiffres entre parenthèses indiquent le recrutement autochtone compris dans le recrutement total. 
RECRUTEMENT TOTAL

(1928 - 1972)

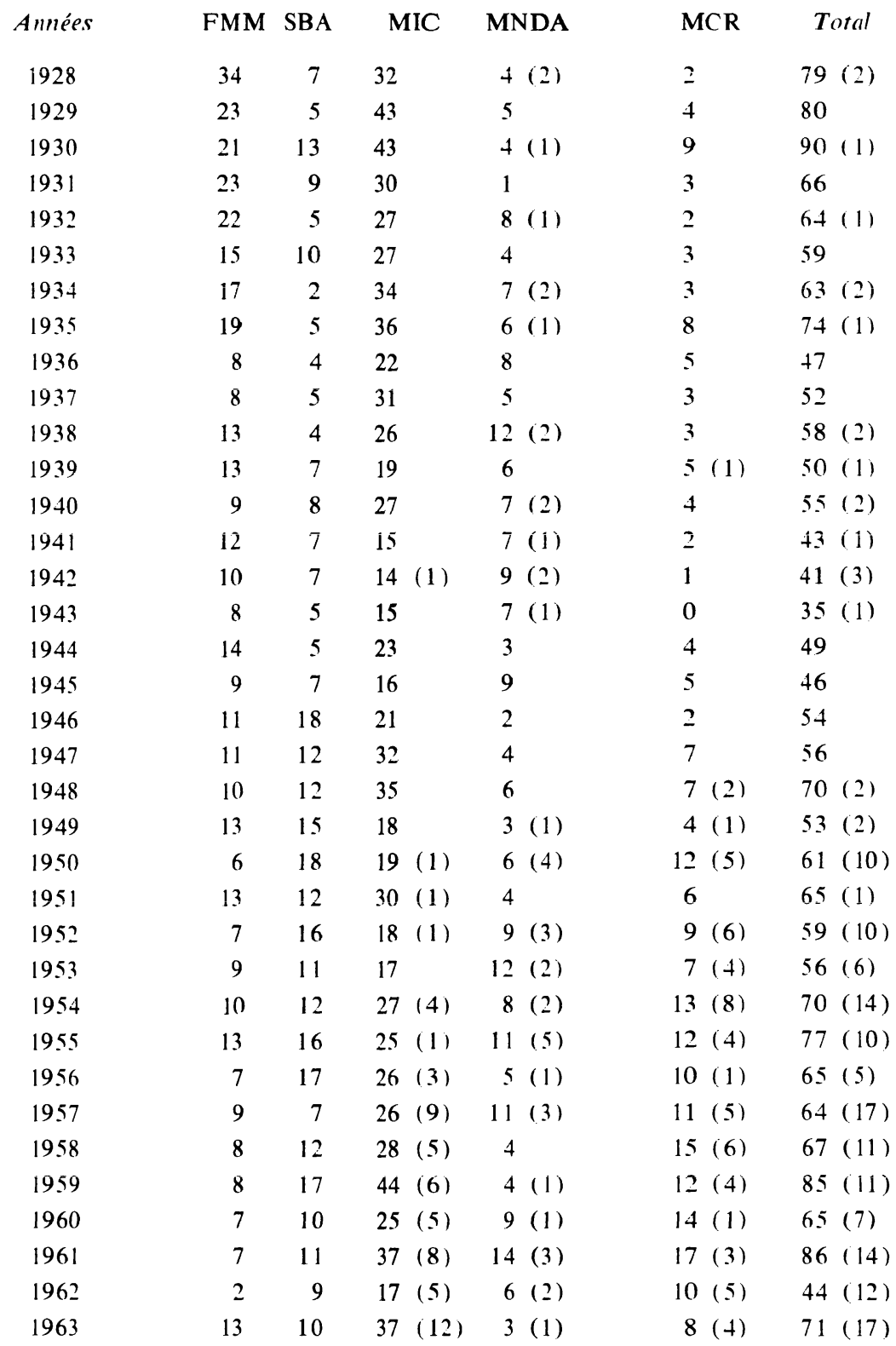


APPENDICE II (suite)

$\begin{array}{ccccccc}\text { Années } & \text { FMM } & \text { SBA } & \text { MIC } & \text { MNDA } & \text { MCR } & \text { Total } \\ 1964 & 4 & 2 & 21(9) & 6(4) & 6(4) & 39(17) \\ 1965 & 3 & 3 & 15(10) & 6(4) & 4(1) & 31(15) \\ 1966 & 2 & 3 & 22(16) & 3(2) & 6(4) & 36(22) \\ 1967 & 5 & 2 & 18(14) & 3(3) & 3(2) & 31(19) \\ 1968 & 4 & 0 & 12(11) & 4(3) & 7(6) & 27(20) \\ 1969 & 0 & 0 & 8(6) & 0 & 2 & 10(6) \\ 1970 & 0 & 0 & 6(5) & 0 & 2(1) & 8(6) \\ 1971 & 0 & 0 & 4(4) & 1(1) & 0 & 5(5) \\ 1972 & 0 & 0 & 6(5) & 1(1) & 3(2) & 10(8)\end{array}$

N.B. Les chiffres entre parenthèses indiquent le recrutement autochtone compris dans le recrutement total. 